\title{
Desarrollo de la inteligencia interpersonal e intrapersonal en educación primaria a partir del uso de tecnologías de información y comunicación: estudio de casos
}

\author{
Eladio Sebastián Heredero ${ }^{1}$ \\ Universidad de Alcalá \\ $\mathrm{M}^{\mathrm{a}}$ del Pilar Garrido Ceballos ${ }^{2}$ \\ (Universidad de Alcalá)
}

\begin{abstract}
Resumen: El desarrollo de las Inteligencias Múltiples es, sin duda, un desafío educativo, cuando para ello incorporamos el uso de las Tecnologías de la Información y la Comunicación al proceso de enseñanzaaprendizaje se propicia, además, la innovación dentro del aula. Hoy en día, las investigaciones avalan que son muchas las ventajas que tiene una utilización adecuada de los recursos TIC, tanto por parte del profesorado como del alumnado, entre ellas destacamos el desarrollo de las diferentes inteligencias intrapersonal e interpersonal. Desarrollo de estas inteligencias y competencia digital son dos objetivos de la educación que pueden y deben caminar juntos. Debemos considerar la competencia digital en la escuela, de forma global e interdisciplinar, no solo como una habilidad en sí misma, sino también como facilitadora de otras habilidades y destrezas necesarias para desenvolverse en la vida cotidiana. Este trabajo pretende responder en qué medida conseguimos desarrollar las inteligencias múltiples, reconocidas hoy en día como integrantes de la inteligencia emocional y consideradas como imprescindibles en la educación integral de los alumnos del siglo XXI mediante el uso de recursos TIC. Palabras clave: Inteligencia interpersonal, Inteligencia intrapersonal, TIC, Innovación en el aula, Competencia digital.
\end{abstract}

Abstract: Development of Multiple intelligences is, without any doubt, an educational challenge; yet including the use of Information and Communication Technologies to the learning process contributes, furthermore, to achieve innovation in the classroom. Nowadays, investigations endorse the many advantages of using suitable ICT resources, both for the teachers and the students, emphasizing the different interpersonal and intrapersonal intelligences. Development of these intelligences and digital abilities are educational two targets that can and must go together. It is a must to consider digital competence in schools, global or interdisciplinary, not only as an ability itself, but also as facilitator of other abilities and skills needed for managing daily life. This research expects to answer the degree of developing multiple intelligences, famous these days as integral part of emotional intelligence and considered essential in student comprehensive education for $21^{\text {st }}$ century, by using ICT resources.

Keywords: Interpersonal intelligence, Intrapersonal intelligence, ICT, Class innovation, Digital competence.

\section{Introducción}

Desde hace algunos años reconocidos técnicos en educación como Tiana (2008) o Ginés (2004) vienen indicando que cada día se hace más necesario que los sistemas educativos se adapten a los cambios de la sociedad y, sobre todo, dotar a los

\footnotetext{
${ }^{1}$ Eladio Sebastián Heredero. Graduado en Magisterio, Licenciado en Ciencias de la Educación por la UCM, Doctor en Educación por la UAH, posdoctorado en Educación por la UNESP (Brasil). Profesor funcionario de Primaria y Secundaria de Castilla la Mancha. Profesor asociado de la UAH. Profesor convidado de la UNESP y UNIARA (Brasil). Especialista en Escuela Inclusiva, Currículo y Organización Escolar.

${ }^{2} \mathrm{M}^{\mathrm{a}}$ del Pilar Garrido Ceballos . Graduada en Magisterio Educación Primaria por la UAM, Graduada en Magisterio de Educación Infantil por la UCJC, Licenciada en Psicopedagogía por la UAM, Máster en Tecnologías para la Función Docente en la Sociedad Multicultural por la UNED, alumna de Doctorado de la UAH. Funcionaria en prácticas por la Consejería de Educación de Castilla la Mancha. Profesora Educación Primaria y Secundaria en centros públicos y concertados de Castilla la Mancha y Madrid.
} 
individuos de las competencias necesarias para desenvolverse con éxito en el mundo del siglo XXI. Se dice en todos los foros educativos que los estudiantes de las escuelas de hoy trabajarán en profesiones que todavía no se han inventado.

En base a ellos, los sistemas educativos pretenden contribuir, desde sus propuestas curriculares, al desarrollo de las capacidades de los alumnos/as, proporcionando una educación de calidad; para conseguir este propósito, las legislaciones educativas incorporan un nuevo elemento a los Currículos: las Competencias Claves.

Pero, además, la neurociencia cuando estudia el complejo funcionamiento cerebral destaca que los procesos de aprendizaje están estrechamente ligados al desarrollo socioafectivo, debido a que el cerebro aprende con mayor efectividad lo que está mediado por la emoción, el sentimiento, y esto potencia aspectos como la atención, la memoria y la percepción. Como dice Goleman (1996) sobre la educación emocional, cuando se trabaja sobre las conductas afectivas se trabaja en el afianzamiento de su personalidad, su autoimagen, su autoconcepto y su autonomía. Esto conlleva que un estudiante motivado, alegre y tranquilo adoptará una actitud más favorable ante los aprendizajes, como indican estudios como los desarrollados por Roger Schank o Martin Seligman.

Los cambios presentes en la sociedad del siglo XXI, en su mayoría, están estrechamente relacionados con las tecnologías debido al gran impacto en el conjunto de la sociedad y, por tanto, también ejerce su influencia en el ámbito de la educación. Los alumnos presentes en nuestras aulas construyen el conocimiento de forma diferente a épocas pasadas. Jukes (2006) propone que los niños del hoy son diferentes debido a su neuroplasticidad; la juventud actual es diferente en cuanto a la forma que piensan, acceden, absorben, interpretan, procesan y usan la información, y en cuanto a la forma en ven, interacciona y se comunican con el mundo actual debido a sus experiencias con las tecnologías digitales.

Santiago y Navaridas (2012) consideran a los educadores, como agentes clave en la transmisión y gestión del conocimiento, y debemos liderar este proceso de transformación educativa, conociendo, aplicando y poniendo en práctica estos nuevos modos de saber, hacer y aprender. Es por ello, que existe una imperante necesidad de cambio en los procesos educativos tradicionales en los que el profesor era el transmisor de la información, del conocimiento, y los discentes meros receptores de dicha información junto con la articulación de emociones y relaciones que nos aporta la neurociencia,

Ante las nuevas exigencias o demandas sociales que cree sujetos más preparados para vivir en este mundo del siglo XXI, es necesario crear modelos educativos innovadores, planteamientos metodológicos distintos que tengan como base un enfoque competencial una participación activa en sus propios procesos de aprendizaje y un planteamiento curricular que priorice una formación integral del sujeto y un desarrollo de su componente socioemocional.

\section{Las competencias clave en la educación del siglo XXI.}

El concepto de competencia ha sido recogido en relevantes trabajos a lo largo de las últimas décadas. Su origen corresponde a las denominadas competencias clave recogidas por la Unión Europea a finales de los 90 del siglo pasado. Por otro lado, en el Informe Delors ("La educación encierra un tesoro", UNESCO, 1996) y en el Proyecto DeSeCo ("Definición y Selección de Competencias", OCDE, 1999-2006), también queda reconocida su importancia. 
El Proyecto DeSeCo, plantea que la adquisición de una competencia por parte de un individuo va más allá de la mera adquisición de conocimientos y destrezas, sino que, incluye destrezas, actitudes y valores necesarios para hacer frente a las demandas complejas en un contexto en particular. El logro de las competencias esenciales en la vida va a permitir el desarrollo sostenible y la cohesión social; de aquí se deriva la importancia de la adquisición de las competencias en el mundo actual.

A través de este proyecto, la OCDE (2005) identifica un conjunto de competencias clave recogidas en tres amplias categorías interrelacionadas:

- Uso y comprensión de herramientas tanto físicas (por ejemplo el uso de la tecnología de la información) como socioculturales (por ejemplo el uso del lenguaje) para interactuar de forma adecuada con el ambiente.

- Interactuar en grupos heterogéneos.

- Actuar de forma autónoma en un contexto social.

Aunque no existe una acepción universal del concepto de competencia, sí se da un cierto acuerdo al considerar las competencias clave como aquellas que debe adquirir todo al alumnado al término de la escolaridad obligatoria puesto que son indispensables para resolver cualquier situación en la vida real y, por tanto, son beneficiosas para los individuos y para la sociedad en general.

Por su parte, la Comisión Europea (2004), establece las competencias claves, entendiéndose éstas como "un paquete multifuncional y transferible de conocimientos, destrezas y actitudes que todos los individuos necesitan para su realización y desarrollo personal, inclusión y empleo".

Una "competencia clave" es crucial para tres aspectos de la vida:

- realización y desarrollo personal a lo largo de la vida (capital cultural);

- inclusión y una ciudadanía activa (capital social);

- aptitud para el empleo (capital humano).

El nuevo marco estratégico para la cooperación europea en el ámbito de la educación y la formación, "Educación y Formación 2020", basado en su antecesor, el programa de trabajo "Educación y Formación 2010", establece los objetivos estratégicos a alcanzar comunes entre los que se incluye: mejorar la calidad y la eficacia de la educación y la formación, es decir, la totalidad de los ciudadanos debe adquirir competencias clave y todos los niveles de educación y formación deben ser más atractivos y eficientes.

Este marco establece objetivos estratégicos comunes para los Estados miembros, junto con una serie de principios para lograrlos, y métodos de trabajo con áreas prioritarias para cada ciclo de trabajo periódico.

De acuerdo con la documentación aportada por la Unión Europea, las políticas educativas se ven en la necesidad de reformular los currículos educativos incorporando las competencias como uno de los ejes vertebradores.

Las competencias clave en el Sistema Educativo Español que aparecen enumeradas y descritas en la Orden ECD/65/2015, de 21 de enero, de aplicación a todo el territorio español reciben la siguiente nomenclatura:

a) Comunicación lingüística.

b) Competencia matemática y competencias básicas en ciencia y tecnología.

c) Competencia digital. 
d) Aprender a aprender.

e) Competencias sociales y cívicas.

f) Sentido de iniciativa y espíritu emprendedor.

g) Conciencia y expresiones culturales.

Centrándonos en nuestro trabajo sabemos que a la hora de integrar las TIC en el proceso de enseñanza- aprendizaje de los estudiantes, no debemos olvidar una serie de aspectos o características de las mismas, que Alfalla, Arena y Median (2001) citados por Marín Díaz (2009) llaman potencialidades, las cuales ayudarán a que la se integren en la práctica de aula de forma armónica. Estos autores señalan nueve:

- Las TIC motivan y estimulan el aprendizaje

- Las TIC tiene flexibilidad para satisfacer las necesidades y capacidades individuales.

- Los ordenadores pueden reducir el riesgo de fracaso en la formación.

- Las TIC dan a los usuarios acceso inmediato a una fuente más rica de información, además de presentarla una nueva forma que ayuda a los usuarios a entenderla y a asimilarla más adecuadamente.

- Las simulaciones por ordenador permiten el pensamiento sistémico sin abandonar la profundidad del análisis. Ideas difíciles se hacen más comprensibles cuando las TIC las hacen visibles.

- Alumnos con profundas y múltiples dificultades de aprendizaje pueden ser motivados a hacer actividades enriquecedoras y formativa pueden incluso compensar las dificultades de comunicación y aprendizaje de usuarios con discapacidades físicas.

- El uso de las TIC hace que los profesores tengan una visión actual sobre cómo enseñar y sobre las formas de aprendizaje.

- Las TIC ofrecen potencial para un trabajo en grupo efectivo.

- Los sistemas de aprendizaje informatizado pueden ayudar a ahorrar dinero y tiempo.

Apoyándonos en estas, podemos afirmar que la utilización de las TIC en el aula de Educación Primaria son un recurso valioso que potenciará además de facilitar y favorecer el desarrollo de las inteligencias inter e intrapersonal y, además ayudarán a informar y formar en los contenidos básicos del currículum, crear guías metodológicas de aprendizaje así como convertirse en un medio de expresión del alumnado.

La integración de las TIC en el currículum de Educación Primaria debe tratar de de cubrir una serie de necesidades de diferente naturaleza, tales como:

- la actualización permanente tanto de los docentes como de los centros escolares,

- el desarrollo del proceso de aprender a aprender en el alumnado,

- transmitir a toda la comunidad social una nueva concepción del término alfabetización.

\section{Las inteligencias interpersonal e intrapersonal}

Todos los humanos poseemos al menos ocho formas de inteligencia, que por lotería genética, se presentan en distintos estados de desarrollo cuando nacemos. Gardner remarca que la brillantez en matemáticas o lengua, las inteligencias más 
favorecidas en el sistema educativo tradicional, no son suficientes para desenvolverse en la vida. Por la naturaleza de nuestro trabajo, nos centramos en dos de ellas:

- Interpersonal: Consiste en la capacidad de entender las intenciones, motivaciones y deseos de otras personas. Permite trabajar y relacionarse de forma efectiva con los demás.

- Intrapersonal: Consiste en la capacidad de entenderse a uno mismo, y apreciar las motivaciones, los sentimientos y los miedos que se sienten. Implica crear un modelo de nosotros mismos que funcione para trabajar de forma efectiva y poder regular nuestras vidas.

Es importante destacar que los alumnos motivados, conscientes de su mejor manera de aprender, progresaran rápido en su aprendizaje. Pero más aún progresarán y madurarán aquellos alumnos que, además, controlen sus emociones, tengan autoestima, adquieran habilidades sociales pertinentes y mantengan empatía con su entorno.

Autores como Mayer y Salovey (1997) y más tarde Goleman (1996) analizarán las inteligencias interpersonales e intrapersonales presentando el constructo de la Inteligencia Emocional importante en sus repercusiones pedagógicas. Gallego, Alonso y Cacheiro (2011, p. 140) recogen los estudios de diferentes psicólogos (Gardner, Sternberg, Salovey o Goleman) que han trabajado este concepto y han detectado cinco áreas o capacidades que la configuran en:

1. Autoconocimiento: Conocer las propias emociones. Tener conciencia de uno mismo.

2. Autocontrol: Controlar las propias emociones. Autorregulación.

3. Automotivación: Motivarse a sí mismo.

4. Empatía: Reconocer las emociones en los demás.

5. Competencia Social: Cuidar las relaciones personales. Contar con habilidades sociales.

\section{Desarrollo de las Inteligencias interpersonal e intrapersonal a través de estrategias metodológicas con TIC}

Desde el ingreso en la escuela del niño hasta su último curso académico, debe sentirse que la esencia de todo proceso educativo es capacitar al individuo para la convivencia y autorrealización y que en estos importantes logros las emociones lejos de ser algo que avergüence o moleste, facilitan el tránsito feliz en el camino hacia la coronación de las metas.

Las emociones juegan un papel decisivo al momento de realizar tareas complejas que requieren tiempo y esfuerzo. Por ello, el gerente debe saber manejar la inteligencia emocional, entendida como un conjunto de habilidades que abarquen la capacidad de motivarse y persistir frente a las decepciones, controlar el impulso y demostrar la gratificación, regular el mal humor, mostrar empatía hacia las demás personas y autoconocer un sentimiento cuando se presenta, variables de tipo psicológico que pudieran obstaculizar el desempeño personal y laboral, así como el logro de colaboración dentro de los equipos de trabajo. Es así como el elemento emocional resulta determinante al introducir innovaciones, particularmente de tipo tecnológico, por la constante adaptación al cambio que implican y la competencia descarnada que pueden desatar.

Entre la teoría de las IM y las TIC podríamos decir que existe un elemento común: la flexibilización. Las IM expresan la existencia de diferentes tipos de inteligencia por lo que la potenciación de una forma única de ellas es un craso error. Y se evidencia, en el fácil manejo de diferentes variables críticas del proceso de 
enseñanza y aprendizaje: por ejemplo el papel que juegan el profesor y los alumnos, así como la configuración de nuevas formas de relacionarnos con la información. No debemos olvidar que la tecnología no debe marcar las metas educativas, pero sí puede ayudar a conseguirlas.

Para conseguir las metas educativas, es necesario poner en juego diferentes estrategias educativas; una de las principales en la que se va a basar este proyecto, por las características individuales de los alumnos, es la estrategia de la enseñanza cooperativa.

Según Sevillano García (2004) considera que "la metodología de enseñanza tiene por objeto la transmisión de los saberes didácticos, de forma dinámica y creadora, al mismo tiempo que favorece el desarrollo de las capacidades humanas, y fomenta actitudes positivas que llevarán al que aprende a su óptima realización personal."

La teoría de las IM estimula a los enseñantes y a sus alumnos a ser imaginativos en la decisión sobre la manera de impartir los contenidos del currículo oficial, y en el establecimiento de métodos para demostrar el conocimiento obtenido por los estudiantes.

En este sentido, juega un papel importantísimo el tipo de aprendizaje que debe estar basado en metodologías activas y por descubrimiento para lograr un aprendizaje significativo. Las nuevas tecnologías exigen un cambio de rol en el profesor y en el alumno

No debemos partir de una enseñanza uniforme puesto que no todas las personas son iguales. Gardner (2001) sostiene que no hay dos personas que tengan exactamente el mismo tipo de mentalidad, puesto que cada una ensambla sus inteligencias con distintas configuraciones.

Como educadores nos enfrentamos a la decisión de ignorar las diferencias o tenerlas en cuenta. Ignorar las diferencias no es justo y existen alternativas; una posibilidad es la educación configurada individualmente, es decir, una educación que tome en serio las diferencias individuales y, en la medida de lo posible, desarrolle prácticas que sirva por igual a los distintos tipos de mentalidades.

El ingrediente esencial es el compromiso para llegar a conocer la mentalidad, la personalidad, de cada estudiante como individuo; es decir, conocer los antecedentes, las virtudes, los intereses, las preferencias, las inquietudes, las experiencias y las metas de cada estudiante, para así garantizar que las decisiones educativas se tomen en función de los perfiles actualizados de los alumnos.

Las estrategias cooperativas son una forma de organización e interacción en el aula que basa en la necesidad de compartir el conocimiento y a través de las cuales, organizando convenientemente los grupos cooperativos, atenderemos los diferentes niveles de aprendizaje que puedan existir y por tanto las diferencias individuales de los alumnos.

Calzadilla (2002) establece que existen al menos tres formas de poner en práctica el aprendizaje colaborativo: la interacción de pares, el tutoreo de pares y el grupo colaborativo (Tudge, 1994). La diferencia entre ellos está determinada por la igualdad en los niveles de rendimiento que exista entre los integrantes. La interacción de pares consiste en la integración de grupos con participantes de diferentes niveles de habilidad, que acometen las ejecuciones en forma organizada y conjunta, participando el docente como mediador y catalizador en las experiencias de aprendizaje del grupo.

El tutoreo de pares (Tudge, 1994), involucra a estudiantes en los que se ha detectado mayor habilidad y a los que se les ha dado un entrenamiento previo para 
servir de coach de sus compañeros de menor nivel, mientras desempeñan el trabajo en forma conjunta; por lo general la interacción entre los estudiantes es tan fluida que logra elevar el nivel de los aprendices y consolidar el que tienen los avanzados, quienes querrán conservar su posición de adelantados y continuarán profundizando en el conocimiento.

Los grupos colaborativos por su parte, tienen mayor tamaño que los primeros y vinculan aprendices de distinto nivel de habilidad, género y procedencia; acumulan el puntaje en forma individual y grupal a lo largo de todo el período, lo que estimula la interdependencia y asegura la preocupación de todos por el aprendizaje de todos, pues el éxito colectivo depende del éxito individual. En este caso el docente debe ser más que un mediador, propiciando un proceso grupal efectivo (Johnson y Johnson, 1992, y Vásquez, Johnson y Johnson, 1993). El grupo puede ser estable o permanente, inestable o circunstancial y de base, que es aquel que va más allá del ámbito académico, desarrollando actividades de soporte y apoyo para el desarrollo integral de sus integrantes.

El aprendizaje colaborativo ha demostrado eficiencia en la superación de actitudes negativas, incrementar la motivación y el autoconcepto; por otra parte las experiencias de interacción cooperativa permiten producir un aprendizaje vinculado al entorno social del individuo, dado que propician la creación de ambientes estimulantes y participativos, en los que los individuos se sienten apoyados y en confianza para consolidar su propio estilo de aprendizaje.

Por su parte, Pere Marqués (2012) establece una serie de razones para aprovechar las posibilidades de innovación metodológica que ofrecen las TIC para lograr una escuela más eficaz e inclusiva como son:

- Alfabetización digital de los alumnos: todos deben adquirir las competencias básicas en el uso de las TIC.

- Productividad: aprovechar las nuevas posibilidades didácticas que ofrecen las ventajas que proporcionan al realizar actividades como: preparar apuntes y ejercicios, buscar información, comunicarnos (e-mail), difundir información...

- Innovar en las prácticas docentes: aprovechar las nuevas posibilidades didácticas que ofrecen las TIC para lograr que los alumnos realicen mejores aprendizajes y reducir el fracaso escolar.

Desde el punto de vista pedagógico, las TIC representan ventajas para el proceso de aprendizaje colaborativo, seguimos las aportaciones de Calzadilla (2002) para exponerlas:

- Estimular la comunicación interpersonal, que es uno de los pilares fundamentales dentro de los entornos de aprendizaje virtual, pues posibilita el intercambio de información y el diálogo y discusión entre todas las personas implicadas en el proceso. En función del diseño del curso, existen herramientas que integran diferentes aplicaciones de comunicación interpersonal o herramientas de comunicación ya existentes (como el correo electrónico o el chat). Estas aplicaciones pueden ser síncronas, como la audio/videoconferencia, las pizarras electrónicas o los espacios virtuales y asíncronos como los foros o listas de discusión.

- Las nuevas tecnologías facilitan el trabajo colaborativo, al permitir que los aprendices compartan información, trabajen con documentos conjuntos y faciliten 
la solución de problemas y toma de decisiones. Algunas utilidades específicas de las herramientas tecnológicas para el aprendizaje cooperativa son: transferencia de ficheros, aplicaciones compartidas, asignación de tareas, calendarios, chat, convocatoria de reuniones, lluvia de ideas, mapas conceptuales, navegación compartida, notas, pizarra compartida, votaciones, etc.

- Seguimiento del progreso del grupo, a nivel individual y colectivo; esta información puede venir a través de los resultados de ejercicios y trabajos, test de autoevaluación y coevaluación, estadística de los itinerarios seguidos en los materiales de aprendizaje, participación de los estudiantes a través de herramientas de comunicación, número de veces que han accedido estos al sistema, tiempo invertido en cada sesión y otros indicadores que se generan automáticamente y que el docente podrá chequear para ponderar el trabajo de cada grupo, pero a su vez los estudiantes podrán también visualizar el trabajo que tanto ellos como el resto de los grupos han efectuado y aplicar a tiempo correctivos y estrategias metacognitivas que tiendan a remediar un desempeño inadecuado.

- Acceso a información y contenidos de aprendizaje: mediante las bases de datos online o bibliográficas, sistemas de información orientados al objeto, libros electrónicos, publicaciones en red, centros de interés, enciclopedias, hipermedias, simulaciones y prácticas tutoriales que permiten a los estudiantes intercambiar direcciones, diversificar recursos e integrar perspectivas múltiples.

- Gestión y administración de los alumnos: permite el acceso a toda aquella información vinculada con el expediente del estudiante e información adicional, que le pueda ser útil al docente en un momento dado, para la integración de grupos o para facilitar su desarrollo y consolidación.

- Creación de ejercicios de evaluación y autoevaluación, con los que el docente podrá conocer el nivel de logro y rediseñar la experiencia de acuerdo a su ritmo y nivel y al estudiante le ofrecerán retroalimentación sobre el nivel de desempeño.

\section{Estudio de casos: Uso de recursos digitales en el desarrollo de la inteligencia intrapersonal e interpersonal en Educación Primaria.}

\subsection{Problema de la investigación. Objetivo.}

El objetivo principal de este trabajo era comprobar que el uso de diferentes recursos TIC en el aula favorece el desarrollo de las inteligencias interpersonal e intrapersonal propuestas por Howard Gardner.

Pensamos que se deben generar nuevos entornos de aprendizaje donde se fomente la creatividad, autonomía y hábitos de trabajo cooperativo entre los estudiantes desde las primeras etapas educativas.

\subsection{Metodología}

\subsubsection{Diseño y muestra}

El presente trabajo se enmarca dentro de un estudio de casos. La muestra seleccionada ha sido un grupo de 24 alumnos de 6 años de edad pertenecientes a un colegio público español. En general el grupo de alumnos presenta una escasa motivación por el aprendizaje y esfuerzo personal. Algunos alumnos presentan baja autoestima y desajustes afectivos que requieren constantemente la ayuda y atención del profesorado. 


\subsubsection{Métodos, técnicas e instrumentos para la recogida de datos}

Se han planteado a los alumnos diferentes actividades educativas a realizar relacionadas con el desarrollo de las IM y el uso de las TIC tal y como se muestra en la tabla 1 y 2.

Inteligencia Intrapersonal Ejemplos de recursos TIC

- Participar en las actividades propuestas con la pizarra digital y con el uso de webs para apoyar los contenidos de las diferentes áreas.

- $\quad$ http://marife.wordpress.com/

- Aplicaciones multimedia para motivar al alumno en el inicio de la lectura, apoyar al proceso lectoescritor y en la lógica-matemática, por ejemplo:

http://www.juntadeandalucia.es/averroes/ 11000642/TIC/pepe/html/menu3.html

http://ocatasus.wix.com/matematicas-infantiles

- Fotografías con cámara digital para trabajar las emociones a través del rostro.

- Audiciones con DVDs y CDs.

- $\quad$ Bits de inteligencia

-http://ntic.educacion.es/w3/recursos/infantil/bits_de_inteligencia/pages/main.htm

Tabla 1. Inteligencia múltiple intrapersonal y recursos TIC utilizados en el aula. Fuente: elaboración propia

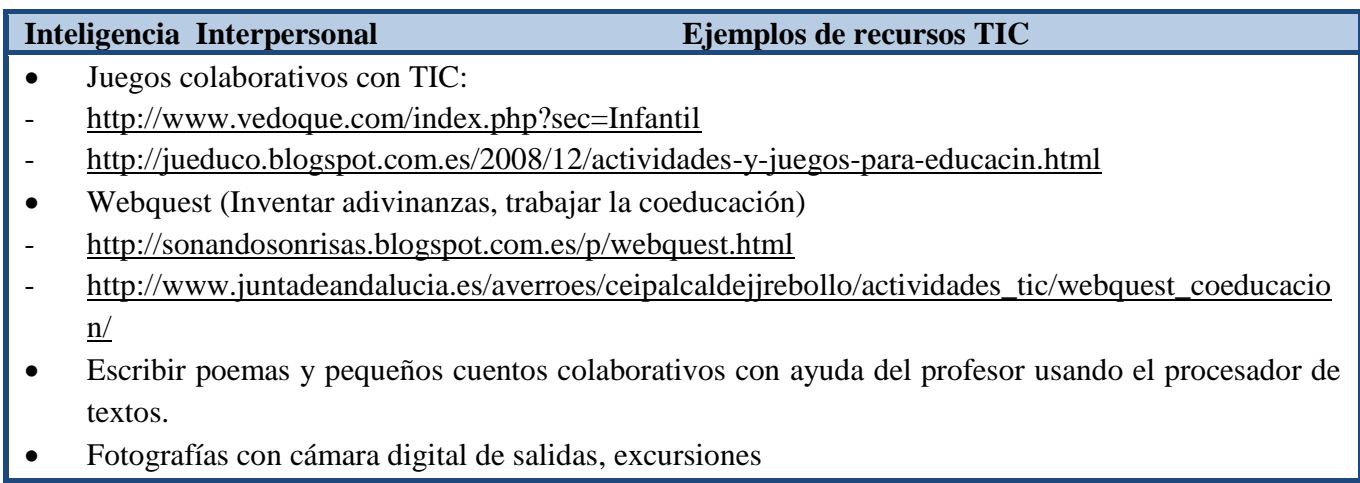

Tabla 2. Inteligencia múltiple interpersonal y recursos TIC utilizados en el aula. Fuente: elaboración propia

La utilización de la observación en nuestra investigación, como procedimiento de recogida de información, nos ha permitido comprender mejor la realidad del aula, de cómo los alumnos se enfrentan a las tareas propuestas con TIC.

Por otro lado, hemos hecho uso de cuestionarios como instrumentos para diagnosticar cómo se encuentra el grupo al inicio y al final de proceso de investigación en relación a las inteligencias interpersonal e intrapersonal.

Los cuestionarios se han tomado del trabajo realizado por Prieto y Ballester (2003) sobre el procedimiento de evaluación de las ocho inteligencias y las estrategias de aplicación por los profesores en el proceso de enseñanza-aprendizaje dentro del aula. Dichos autores hicieron una adaptación del Proyecto Spectrum de Gardner, Feldman, Krechevsky (2000); estos últimos, en sus trabajos de investigación elaboraron una lista con dieciocho características mientras los alumnos realizaban actividades en los distintos dominios.

Para nuestra investigación hemos adaptado dos de los inventarios de evaluación de Prieto y Ballester (2003) a la etapa de educación básica. Se trata de dos inventarios, uno para cada tipo de inteligencia. Los inventarios están formados por 6 ítems en una escala tipo Likert de 1 a 4 , permitiendo evaluar un grado mayor que la simple presencia o ausencia de la habilidad o característica en el alumnos. Además, se 
añade un apartado "Observaciones" en la que el maestro puede realizar cualquier anotación que considere oportuna para la evaluación de cada inteligencia. Su aplicación la ha realizado el tutor a cada uno de los alumnos del grupo de forma individualizada.

\subsection{Resultados.}

El uso de las TIC en el aula ha provocado una renovación didáctica en el aula, donde se ha puesto en práctica una metodología activa e innovadora favoreciendo el interés de los alumnos por el aprendizaje y por tanto el desarrollo de las inteligencias múltiples inter e intrapersonal.

Realizando una comparativa entre el inicio y el fin de la investigación, podemos constatar, que se ha producido un aumento, un avance en cada una de las inteligencias. Hemos realizado un análisis detallado de la inteligencia interpersonal e intrapersonal, ya que nos que nos interesa comprobar en qué medida se han cumplido el objetivo propuesto en la investigación, es decir, si la utilización de los recursos TIC favorece el desarrollo de estas inteligencias. Para el tratamiento de los datos y realizar un análisis comparativo, se ha utilizado el programa estadístico SPSS versión 20.

\begin{tabular}{|c|c|c|c|c|c|c|}
\hline \multicolumn{7}{|c|}{ Inteligencia intrapersonal. Estadísticos inicio de la investigación. } \\
\hline Ítems & \multicolumn{2}{|c|}{\begin{tabular}{|l|l}
1. & \\
\end{tabular}} & 4. & 5. & 7. & 8. \\
\hline Válidos & \multicolumn{2}{|l|}{24} & 24 & 24 & 24 & 24 \\
\hline Perdidos & \multicolumn{2}{|l|}{0} & 0 & 0 & 0 & 0 \\
\hline Media & \multicolumn{2}{|c|}{3,29} & 2,08 & 2,54 & 2,25 & 3,21 \\
\hline Desv. típ. & \multicolumn{2}{|c|}{,751 } & ,881 & 977 &, 532 &, 721 \\
\hline \multirow{6}{*}{$\begin{array}{l}\text { Relación de } \\
\text { ítems }\end{array}$} & 1. & \multicolumn{5}{|c|}{ Es consciente de sus emociones (cariño, rabia, ira...) } \\
\hline & 3. & \multicolumn{5}{|c|}{ Se desenvuelve bien cuando se le deja trabajar con autonomía } \\
\hline & 4. & \multicolumn{5}{|c|}{ Manifiesta curiosidad por los interrogantes de la vida } \\
\hline & 5. & \multicolumn{5}{|c|}{ Su motivación le lleva a cumplir y lograr lo que se propone } \\
\hline & 7. & \multicolumn{5}{|c|}{ Prefiere trabajar de forma individual } \\
\hline & 8. & \multicolumn{5}{|c|}{ Sabe expresar cómo se siente (enfado, alegría...) } \\
\hline
\end{tabular}

Tabla 3. Inteligencia intrapersonal. Estadísticos inicio de la investigación Fuente: elaboración propia

\begin{tabular}{|c|c|c|c|c|c|c|}
\hline \multicolumn{7}{|c|}{ Inteligencia intrapersonal. Estadísticos final de la investigación. } \\
\hline Ítems & 1. & \begin{tabular}{l|l|}
3. &
\end{tabular} & 4. & 5. & 7. & 8. \\
\hline Válidos & 24 & 24 & 24 & 24 & 24 & 24 \\
\hline Perdidos & 0 & 0 & 0 & 0 & 0 & 0 \\
\hline Media & 3,63 & 3,04 & 2,38 & 3,08 & 2,08 & 3,63 \\
\hline Desv. típ. & ,495 & ,859 &, 875 & ,830 & , 408 &., 576 \\
\hline \multirow{6}{*}{$\begin{array}{l}\text { Relación de } \\
\text { ítems }\end{array}$} & 1 & \multicolumn{5}{|c|}{ Es consciente de sus emociones (cariño, rabia, ira...) } \\
\hline & 3 & \multicolumn{5}{|c|}{ Se desenvuelve bien cuando se le deja trabajar con autonomía } \\
\hline & 4 & \multicolumn{5}{|c|}{ Manifiesta curiosidad por los interrogantes de la vida } \\
\hline & 5 & \multicolumn{5}{|c|}{ Su motivación le lleva a cumplir y lograr lo que se propone } \\
\hline & 7 & \multicolumn{5}{|c|}{ Prefiere trabajar de forma individual } \\
\hline & 8 & \multicolumn{5}{|c|}{ Sabe expresar cómo se siente (enfado, alegría...) } \\
\hline
\end{tabular}

Tabla 4. Inteligencia intrapersonal. Estadísticos final de la investigación Fuente: elaboración propia 
Observando los datos que presentan las tablas 3 y 4 podemos decir que las puntuaciones medias de los todos los ítems han subido excepto en el caso del ítem 7 , por lo que deducimos que después de incluir las TIC dentro de los recursos para el desarrollo de actividades los alumnos reconocen un mayor número de veces sus emociones, han mejorado su autonomía a la hora de enfrentarse a una tarea determinada, presentan más curiosidad por los aspectos de la vida han aumentado su motivación y ha aumentado el espíritu de colaboración entre iguales saben expresar mejor sus sentimientos.

\begin{tabular}{|c|c|c|c|c|c|c|c|}
\hline \multicolumn{8}{|c|}{ Inteligencia interpersonal. Estadísticos inicio de la investigación. } \\
\hline \multicolumn{2}{|c|}{ Ítems } & \multirow{2}{*}{24} & \multirow{2}{*}{\begin{tabular}{l|l|} 
& 4. \\
& 24
\end{tabular}} & \multirow{2}{*}{$\begin{array}{ll}7 . \\
24\end{array}$} & \multirow{2}{*}{\begin{tabular}{|l|}
8. \\
24
\end{tabular}} & \multirow{2}{*}{\begin{tabular}{|l|}
9. \\
24
\end{tabular}} & \multirow{2}{*}{10.} \\
\hline Válidos & 24 & & & & & & \\
\hline Perdidos & 0 & 0 & 0 & 0 & 0 & 0 & 0 \\
\hline \multicolumn{2}{|l|}{ Media } & 3,21 & 2,67 & 2,17 & 3,00 & 2,54 & 2,54 \\
\hline \multicolumn{2}{|l|}{ Desv. típ. } & 779 & \begin{tabular}{l|l|}
, 816 \\
\end{tabular} & ,816 & 722 & 833 &, 884 \\
\hline \multirow{6}{*}{$\begin{array}{l}\text { Relación } \\
\text { de ítems }\end{array}$} & & 1. & \multicolumn{5}{|c|}{$\begin{array}{l}\text { Le gusta relacionarse con sus compañeros y amigos. Se } \\
\text { muestra sociable con ellos }\end{array}$} \\
\hline & & 4. & \multicolumn{5}{|c|}{ Se maneja muy bien en diversos ámbitos } \\
\hline & & 7. & \multicolumn{5}{|c|}{$\begin{array}{l}\text { Muestra habilidades para mediar cuando algunos amigos } \\
\text { tienen conflictos }\end{array}$} \\
\hline & & 8. & \multicolumn{5}{|c|}{$\begin{array}{l}\text { Es flexible. Se adapta bien a diferentes situaciones y grupos } \\
\text { de personas }\end{array}$} \\
\hline & & 9. & \multicolumn{5}{|c|}{ Establece empatía con facilidad y se preocupa por los demás } \\
\hline & & 10. & \multicolumn{5}{|c|}{ Los otros buscan su compañía } \\
\hline
\end{tabular}

Tabla 5. Inteligencia interpersonal. Estadísticos inicio de la investigación Fuente: elaboración propia

\begin{tabular}{|c|c|c|c|c|c|c|c|}
\hline \multicolumn{8}{|c|}{ Inteligencia interpersonal. Estadísticos final de la investigación. } \\
\hline \multicolumn{2}{|l|}{ Ítems } & \multirow{2}{*}{1.} & \multirow{2}{*}{\begin{tabular}{|l|}
4. \\
24
\end{tabular}} & \multirow{2}{*}{$\begin{array}{ll}7 . \\
24\end{array}$} & \multirow{2}{*}{\begin{tabular}{|l|}
8. \\
24 \\
\end{tabular}} & \multirow{2}{*}{$\begin{array}{ll}9 . \\
24\end{array}$} & \multirow{2}{*}{$\frac{10 .}{24}$} \\
\hline Válidos & 24 & & & & & & \\
\hline Perdidos & 0 & 0 & 0 & 0 & 0 & 0 & 0 \\
\hline \multicolumn{2}{|l|}{ Media } & 3,54 & 2,92 & 2,75 & 3,25 & 2,88 & 3,04 \\
\hline \multicolumn{2}{|l|}{ Desv. típ. } &, 588 &, 776 & 944 &, 737 & 900 &, 806 \\
\hline \multirow{6}{*}{$\begin{array}{l}\text { Relación } \\
\text { de ítems }\end{array}$} & & 1. & \multicolumn{5}{|c|}{$\begin{array}{l}\text { Le gusta relacionarse con sus compañeros y amigos. Se muestra } \\
\text { sociable con ellos }\end{array}$} \\
\hline & & 4. & \multicolumn{5}{|c|}{ Se maneja muy bien en diversos ámbitos } \\
\hline & & 7. & \multicolumn{5}{|c|}{$\begin{array}{l}\text { Muestra habilidades para mediar cuando algunos amigos tienen } \\
\text { conflictos }\end{array}$} \\
\hline & & 8. & \multicolumn{5}{|c|}{$\begin{array}{l}\text { Es flexible. Se adapta bien a diferentes situaciones y grupos de } \\
\text { personas }\end{array}$} \\
\hline & & 9. & \multicolumn{5}{|c|}{ Establece empatía con facilidad y se preocupa por los demás } \\
\hline & & 10. & \multicolumn{5}{|c|}{ Los otros buscan su compañía } \\
\hline
\end{tabular}

Tabla 6. Inteligencia interpersonal. Estadísticos final de la investigación Fuente: elaboración propia 
Observando los datos de las tablas 5 y 6 podemos decir que las puntuaciones medias de los todos los ítems han subido, por lo que deducimos que después incluir las TIC entre los recursos para el desarrollo de actividades colaborativas, y se empiezan a aceptar más con sus diferencias individuales. Por otro lado, señalar que el grado de dispersión se ha reducido en todos los ítems. De su interpretación realizamos el siguiente análisis: los alumnos se muestran más sociables a la hora de relacionarse, se manejan cada vez mejor en diferentes situaciones, intentan mediar más cuando hay conflictos o buscan la ayuda del profesor para solucionarlos, existe una mayor adaptación al grupo, están más integrados, el clima de aula ha mejorado, son un poco más empáticos entre ellos y por eso han disminuido los conflictos, se suelen relacionar más.

\section{Conclusiones}

Vemos, en el ámbito educativo, como las transformaciones sociales y tecnológicas sufridas en estos últimos años han sido importantísimas. El panorama actual, de un lado, fundamentado en la necesidad de reflexionar sobre lo que es necesario para el ciudadano de hoy en día y, de otro, debido a los cambios tecnológicos, hace necesaria poner en juego metodologías que favorezcan la adquisición de las competencias clave.

Atendiendo a esta investigación y teniendo en cuenta los datos recogidos a través de la observación participante y el análisis de los cuestionarios al inicio y final del proceso, hemos podido comprobar que efectivamente la planificación e inclusión progresiva de los medios TIC han potenciado el desarrollo de las IM.

Concretamente en lo que se refiere a la inteligencia intrapersonal observamos que los alumnos después de trabajar con las TICs son más conscientes de sus emociones (cariño, rabia, ira...); dicen que se desenvuelven bien cuando se le deja trabajar con autonomía; manifiestan más curiosidad por los interrogantes de la vida; su motivación les lleva a cumplir y lograr lo que se proponen y, además, sabe expresar cómo se siente (enfado, alegría...). Se constata también que pierde interés por trabajar de forma individual. Los cambios producidos, aun considerando la relatividad de la investigación por las características de la muestra, parece que indican un cambio a mejor en esta inteligencia, es decir, se desarrolla con las TIC.

En lo referente a la inteligencia interpersonal, los datos son similares tras el uso de las TICs en el aula en la muestra y condiciones descritas. Así vemos que les gusta relacionarse más con sus compañeros y amigos y que se muestran más sociables con ellos; dicen que se manejan mejor en diversos ámbitos; muestran habilidades para mediar cuando algunos amigos tienen conflictos; tienen mayor flexibilidad; se adaptan mejor a diferentes situaciones y grupos de personas e, indican que establecen empatía con más facilidad y se preocupan más por los demás y, además, manifiestan que los otros buscan más su compañía.

Pensamos que para que el uso de la tecnología educativa sea fructífero se requiere su correcto uso e inclusión en los procesos educativos, por un lado se hace necesario que la administración dote a los centros educativos de instalaciones y recursos convenientes y, por otro, la necesidad de un perfil de profesor innovador y formado en el conocimiento y uso de las TIC para mejorar la calidad del proceso de enseñanza-aprendizaje desde un enfoque competencial, se debe hacer hincapié en la importancia del principio de actividad, de participación activa de los propios estudiantes en su proceso de aprendizaje para que adquieran destrezas y capacidades que les permitan desenvolverse con éxito en el mundo actual. 
Coincidimos con Marín y Sampedro (2016), y queremos dejar constancia, de que tanto las familias como la comunidad consumen medios tecnológicos en su vida diaria como elementos para la comunicación y la interacción social; las herramientas TIC se convierten, de esta manera, en instrumentos esenciales para la participación en la sociedad, igualmente como elementos de dinamización para su intervención y cooperación en el entorno escolar.

\section{Referencias bibliográficas}

Calzadilla, M (2002). Aprendizaje colaborativo y Tecnologías de la Información y la Comunicación. OEI-Revista Iberoamericana de Educación. Recuperado de: $<$ http://www.procesosemivirtual-

ese.com/mat/proy investigacion protocolo/COLIN GONZALEZ MONSERRAT M INUI/texto/2-El\%20Aprendizaje\%20con\%20las\%20TICs.pdf > [Consultado el 12 de febrero de 2015]

Comisión Europea. Dirección General de Cultura y Educación (2004). Puesta en práctica del programa de trabajo "Educación y Formación 2010". Competencias clave para un aprendizaje a lo largo de la vida. Un marco europeo de referencia. Recuperado de: $\quad<$ http://www.colombiaaprende.edu.co/html/productos/1685/articles174000_clave.pdf > [Consultado el 12 de febrero de 2015]

Delors, J. (1996). Informe de la UNESCO de la Comisión Internacional sobre la educación para el siglo XXI. La educación encierra un tesoro. Recuperado de: $\langle$ http://www.servicios.uns.edu.ar/institucion/files/106_AV_1.pdf $>$ [Consultado el 12 de febrero de 2015]

España. (2015). Orden ECD/65/2015, de 21 de enero, por la que se describen las relaciones entre las competencias, los contenidos y los criterios de evaluación de la educación primaria, la educación secundaria obligatoria y el bachillerato. BOE de 22/01/2015.

Gallego, D. J., Alonso, C. M. y Cacheiro, M. L. (Coords.) (2011). Educación, sociedad y tecnología. Madrid. Editorial Ramón Areces

Gardner, H. (2001). La inteligencia reformulada: las inteligencias múltiples del siglo $X X I$. Barcelona. Paidós Ibérica.

Ginés Mora, J. (2004) La necesidad del cambio educativo para la sociedad del conocimiento. Rev. Iberoamericana de Educación. OEI. n. 35, may/ago 2004. Recuperado de: 〈http://rieoei.org/rie35a01.htm> [Consultado el 12 de febrero de 2015].

Goleman, D. (1996) La inteligencia Emocional. Barcelona: Ed. Kairós.

Jukes, I. y Dosaj, A. (2006). Understanding Digital Children (DKs). Teaching \& Learning in the New Digital Landscape. Recuperado de: $<$ https://www.ibo.org/ibap/conference/documents/IanJukes-

UnderstandingDigitalKids.pdf $>$ [Consultado el 12 de febrero de 2015]

Marco Estratégico Educación y Formación 2020 (2012). Recuperado de: http://www.mecd.gob.es/educacion-mecd/mc/redie-eurydice/prioridades-

europeas/et2020.html[Consultado el 12 de febrero de 2015] 
Marín Díaz, V. (Coord.), (2009). Las TIC y el desarrollo de las competencias básicas. Una propuesta para Educación Primaria. Barcelona. MAD

Marín Díaz, V. y Sampedro Requena, B. E. (2016).Web 2.0 for the invigoration and participation of families and communities. Journal of New Approaches in Educational Research, 5(1), 38-43. doi: 10.7821/naer.2016.1.137 Recuperado de: <http://naerjournal.ua.es/article/view/v5n1-6> [Consultado el 12 de febrero de 2015]

Marqués, P. (2012). Impacto de las TIC en la educación: funciones y limitaciones. Revista de Investigación Editada por Área de Innovación y Desarrollo, SL. Recuperado de: <http://www.3ciencias.com/wp-content/uploads/2013/01/impacto-delas-tic.pdf > [Consultado el 12 de febrero de 2015]

MECD (2012) Marco Estratégico Educación y Formación 2020. Recuperado de: $<$ http://www.mecd.gob.es/educacion-mecd/mc/redie-eurydice/prioridadeseuropeas/et2020.html > [Consultado el 12 de febrero de 2015]

OCDE (2005): La definición y selección de competencias clave. Resumen Ejecutivo. Recuperado de: <http://www.deseco.admin.ch/bfs/deseco/en/index/03/02.parsys.78532.downloadList. 94248.DownloadFile.tmp/2005.dscexecutivesummary.sp.pdf $>$ [Consultado el 12 de febrero de 2015].

Prieto, M.D. y Ballester, P. (2003). Las inteligencias múltiples: diferentes formas de enseñar y aprender. Madrid. Pirámide.

Santiago y Navaridas (2012). En la Web 2.0. en escena. Pixel-Bit: Revista de medios y educación, ISSN 1133-8482, no 41, (pág.19-30). Recuperado de:

<http://www.redalyc.org/articulo.oa?id=36828247002> [Consultado el $12 \mathrm{de}$ febrero de 2015]

Sevillano García, M.L. (2004). Estrategias innovadoras para una enseñanza de calidad. Madrid. Pearson.

Tiana Ferrer, A. (2008). Evaluación y Cambio de los Sistemas Educativos: la Interacción que hace falta. Rev. Ensaio: avaliação de políticas públicas em Educação. Rio de Janeiro, v. 16, n. 59, p. 275-296, abr./jun. 2008. Recuperado de:

$<$ http://www.scielo.br/pdf/ensaio/v16n59/v16n59a07.pdf> [Consultado el 12 de febrero de 2015] 